\title{
A case of calciphylaxis and acute myeloid leukemia: A previously unreported association
}

\author{
Joshua D. Rosenberg ${ }^{1^{*}}$, Pamela Boswell ${ }^{2}$, William Miller ${ }^{1}$ \\ ${ }^{1}$ Division of Hematology/Oncology, Scripps Green Clinic, La Jolla, USA; \\ *Corresponding Author: rosenberg.joshua@scrippshealth.org \\ ${ }^{2}$ Division of Hematopathology, Scripps Clinic Medical Group, La Jolla, USA
}

Received 1 March 2013; revised 2 April 2013; accepted 10 May 2013

Copyright (C) 2013 Joshua D. Rosenberg et al. This is an open access article distributed under the Creative Commons Attribution License, which permits unrestricted use, distribution, and reproduction in any medium, provided the original work is properly cited.

\begin{abstract}
Calciphylaxis is a condition of induced hypersensitivity in which tissues respond to challenge agents with local calcification. This article reports the first known case of diffuse calciphylaxis associated with acute myeloid leukemia resulting in death from hypoxic respiratory failure and refractory hypotension.
\end{abstract}

Keywords: Calciphylaxis; Acute Myeloid Leukemia; Respiratory Failure; Hypotension

\section{INTRODUCTION}

Calciphylaxis is a rare, poorly understood vasculopathic syndrome characterized by ischemia, thrombosis and necrosis due to calcification. Multiple sensitizing agents and subsequent challengers associated with calciphylaxis have previously been described. Prior case reports have reported the association of specific malignancies with calciphylaxis including metastatic breast cancer, multiple myeloma, Hodgkin's lymphoma, chronic myelomonocytic leukemia, cholangiocarcinoma and malignnant melanoma. We present a case of newly diagnosed, acute myeloid leukemia with fatal hypoxic respiratory failure and refractory hypotension felt secondary to what was subsequently identified as diffuse calciphylaxis involving multiple organ systems. Clinicians caring for patients with acute myeloid leukemia should be aware of the possible association with diffuse, systemic calciphylaxis and consider this entity in patients with refractory hypotension and respiratory failure with no clear explanation. Though no therapeutic modality is clearly superior in treating calciphylaxis, treatments do exist that may limit and potentially reverse the damage caused by this disease process.

\section{CASE PRESENTATION}

A 46 years old female with a past medical history of hypertension presented to an outside institution with a five-day history of flu-like symptoms and neck discomfort. In the emergency room the patient was febrile, hypotensive $(62 / 44 \mathrm{mmHg})$ and had evidence of acute kidney injury (creatinine $4.7 \mathrm{mg} / \mathrm{dL}$; reference $0.6-1.5$ $\mathrm{mg} / \mathrm{dL}$ ) with a urinalysis suggesting urosepsis as the source of infection given a positive leukocyte esterase and nitrite component. The patient ultimately required norepinephrine blood pressure support as she was refractory to intravenous fluid resuscitation. A computed tomography (CT) scan of the abdomen and pelvis revealed a non-obstructive, 5 millimeter calculus in the left pelvis/proximal ureter, hepatomegaly and a prominent spleen. The patient was placed on antibiotics and taken for extracorporeal shock wave lithotripsy of the left proximal ureteral calculus with left ureter stent placement.

Furthermore, labs obtained upon presentation included a macrocytic anemia (hemoglobin $10.3 \mathrm{~g} / \mathrm{dL}$; reference range $12.0-16.0 \mathrm{~g} / \mathrm{dL}$ and mean corpuscular volume $102.7 \mathrm{fl}$; reference range $80-100 \mathrm{fl})$ and thrombocytopenia $(76 \mathrm{~K} / \mathrm{mcL}$; reference range $150-450 \mathrm{~K} / \mathrm{mcL})$ with normal white blood cell count and differential. Initial work up included a peripheral blood smear that demonstrated no schistocytes or leukoerythroblastic changes. Biochemical studies ruled out iron or vitamin deficiencies. At this juncture, the patient's blood count abnormalities were felt to most likely reflect an underlying infection although there was no culture proven organism.

The patient initially responded to antibiotics and fluid resuscitation with a significant correction in her kidney function. However, she was found to have progressive thrombocytopenia and leukocytosis with a white blood cell count up to $21 \mathrm{~K} / \mathrm{mL}$. A sternal bone marrow aspira- 
tion was performed that revealed acute myeloid leukemia. She had a normal, female karyotype. Polymerase chain reaction demonstrated an insertion mutation in exon 12 of the nucleophosmin gene while demonstrating no mutations for FMS-like tyrosine kinase 3 and normal CEBPA mutation screen.

In conjunction with this diagnosis, the patient was found to have increasing neck discomfort associated with dysphagia. A CT scan of the neck revealed a 7 centimeter retropharyngeal abnormality felt to represent a fluid collection or abscess with infraglottic airway narrowing secondary to bilateral thyroid enlargement and lymphadenopathy. She was evaluated by a head and neck surgeon who felt this lesion to be secondary to leukemic infiltration as opposed to an infection. Therefore, incision and drainage was felt to be unnecessary. Soon thereafter, with subjective difficulty breathing, the patient was intubated prophylactically given the fear of evolving airway obstruction.

The patient was transferred to our hospital intubated, sedated and on broad spectrum antibiotics awaiting further management of her leukemia. At this point, she was still febrile and hypotensive requiring pressor support although all cultures remained negative. Her kidney function remained in the $1.5-1.6 \mathrm{mg} / \mathrm{dL}$ range throughout her stay at our facility. A repeat CT scan demonstrated a 10 centimeter bilateral neck mass arising from the thyroid and surrounding the trachea and esophagus (Figure 1). Additionally, the patient had small, bilateral, scattered lymphadenopathy in the cervical, supraclavicular, and mediastinal regions with an opacification in the right lower lung zone. No calcifications were seen on imaging. A fine needle aspiration of her neck mass demonstrated fragmented skeletal muscle with infiltration by an immature myeloid neoplasm and no calciphylaxis. Analysis by flow cytometry revealed a population

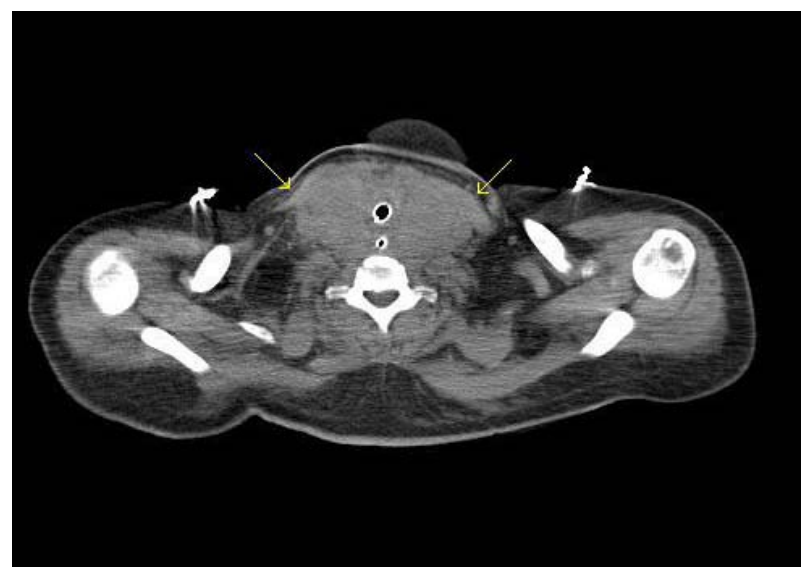

Figure 1. CT neck, arrows point to $10 \mathrm{~cm}$ bilateral neck mass involving the thyroid gland, surrounding the trachea and esophagus. The mass has a soft tissue density similar to muscle, with no gross calcifications identified. of blasts with the same immunophenotype as previously identified in the peripheral blood and bone marrow, consistent with a myeloid sarcoma.

The patient was started on remission induction chemotherapy utilizing idarubicin and cytarabine. Though she was on broad spectrum anti-bacterial, anti-fungal and anti-viral therapy she required increasing pressor support during her hospitalization. Imaging suggested a consolidative pneumonia although no organisms were identified on successive bronchospies with bronchoalveloar lavage. Furthermore, the patient's pulmonary status continued to decline with worsening hypoxemic respiratory failure. She ultimately passed away after her durable power of attorney made the patient comfort care only. A postmortem autopsy revealed diffuse calciphylaxis involving the lungs, scalp, subcutaneous soft tissues, peri-thyroid soft tissue and liver (Figures 2-4).

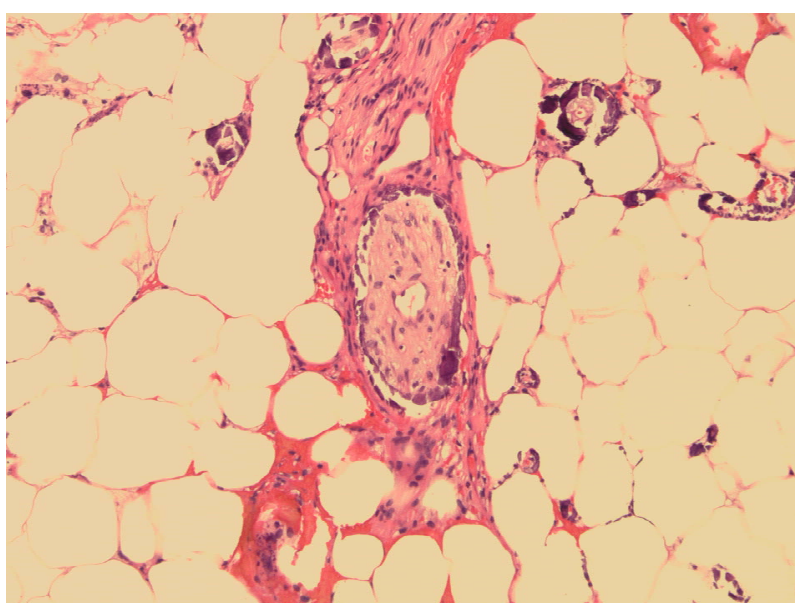

Figure 2. Scalp biopsy, showing subcutaneous soft tissue with calcifications, including perineural involvement. H \& E (Hematoxylin Eosin) staining, 20× original magnification.

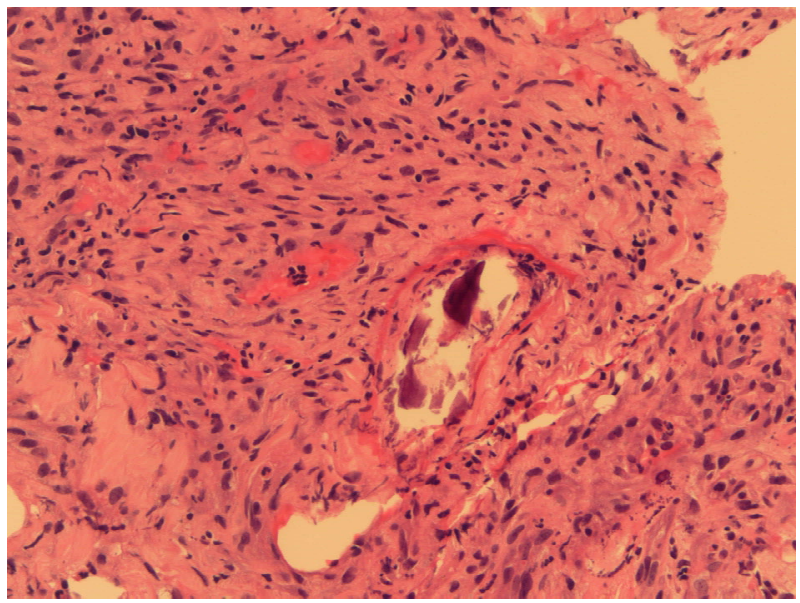

Figure 3. Lung biopsy, showing calcifications enveloped by dense inflammation. H \& E (Hematoxylin Eosin) staining, 40× original magnification. 


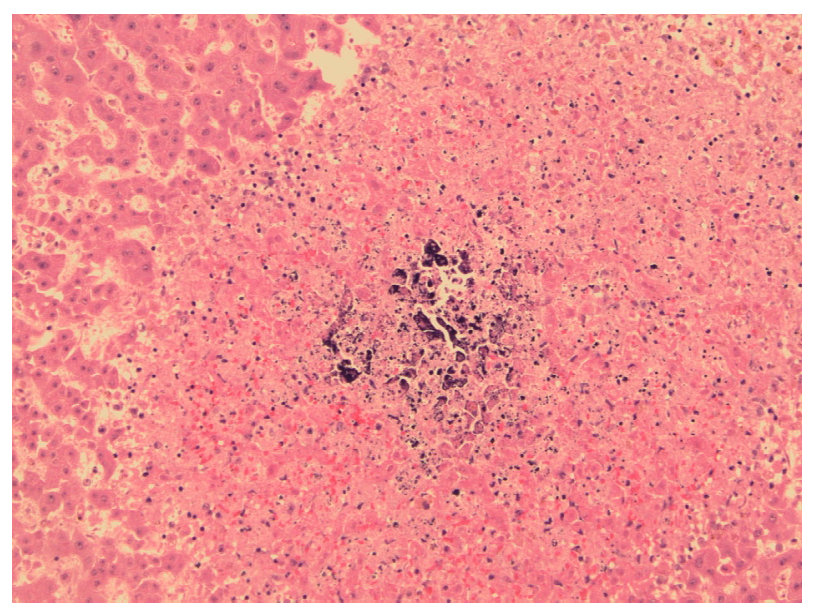

Figure 4. Liver biopsy, showing patchy calcifications which were present throughout the liver. H \& E (Hematoxylin Eosin) staining, 20× original magnification.

\section{DISCUSSION}

In 1962, Hans Selye originally defined calciphylaxis as a condition of induced hypersensitivity in which tissues responded to challenging agents with local calcifycation [1]. Selye demonstrated that systemic sensitizing agents such as dihydrotachysterol, vitamin D2, vitamin D3, parathyroid hormone, and sodium acetylsulfathizole followed by exposure to a "challenger" (e.g., metallic salts such as iron, chromium, aluminum, manganese, titanium, lead, organic compounds such as egg albumin or trauma) could induce calcification of various organs including the skin [1-4]. After Selye's definition, other terms, including calcific uremic arteriolopathy [5], calcific small-vessel ischemic disease [6], uremic gangrene syndrome [7] and vascular calcification-cutaneous necrosis syndrome [8], have been proposed to describe this clinical entity. However, for the human syndrome, calciphylaxis is still the most commonly used term. The diagnosis is made on the basis of pathologic findings including me- dial arteriolar calcification, subintimal fibrosis, arterial thrombosis and ischemic cutaneous necrosis with the correct clinical picture and absence of vasculitic changes.

The precise pathogenesis of calciphylaxis is not clear. A recent review aiming to construct a pathogenic pathway looked at regulatory receptors and ligands that affect bone mineralization and resorption and how these could be affected by risk factors previously associated with calciphylaxis. The author felt that the final common pathway leading to vascular calcification was nuclear factor к-B (NFKB) activation and that "sensitizers" and "challengers" would directly or indirectly activate this pathway. The receptor activator of NFKB (RANK) and its ligand (RANKL) are essential for normal bone development and bone mineral resorption. High NFKB ac- tivity is associated with bone mineral loss. Conversely, osteoprotegerin (OPG) is an antaganost of RANK ligand, thereby inhibiting the RANK pathway. Increased expression of RANKL or deficient OPG may cause osteopenia, fractures and vascular calcification. The author felt that the etiology of malignancy induced extraosseous calcifycation could be secondary to the following: 1) tumor production of PTH or PTH-related peptide, vitamin D or various cytokines; 2) up-regulation of RANKL; 3) downregulation of OPG or potentially a combination of all of the above [9].

Kidney failure is the most important risk factor associated with the development of calciphylaxis as illustrated in a single institution cohort of 64 patients with calciphylaxis revealing $77 \%$ to be receiving dialysis for end-stage renal failure [10]. However, it has also been reported in patients with normal renal and parathyroid function, so called, "non-traditional" patients [11]. In this review, $27.8 \%$ of cases were caused by primary hyperparathyroidism. This was followed by malignancy (22.2\%), alcoholic liver disease (16.7\%), connective tissue disease $(11.1 \%)$, chemotherapy-induced protein C and $\mathrm{S}$ deficiency and chronic kidney disease, though not end-stage kidney disease $(2.8 \%)$, respectively. Previous case reports have reported the association of specific malignancies with calciphylaxis including metastatic breast [12], multiple myeloma [13], Hodgkin's lymphoma [14], chronic myelomonocytic leukemia [15], cholangiocarcinoma [16] and malignant melanoma [17]. Additional risk factors associated with the development of calciphylaxis have included an elevated calcium-phosphate product, female sex, obesity, Caucasian race and hypoalbuminemia [18]. This paper also discusses the possibility of ischemia induced cellular injury provoking smooth muscle cells of vascular tissue to dedifferentiate in osteoblast-like cells. These cells can produce bone matrix proteins that create calcification in vitro. One of these proteins, osteopontin, has been found at the base of calcium aggregates in calcified vessels from calciphylaxis patients and not in noncalcified vessels from the same patient.

As outlined in a review article published in the Archives of Dermatology, several factors have been statistically associated with developing calciphylaxis in addition to those mentioned above, including liver disease, systemic corticosteroid use, increased erythrocyte sedimentation rate, elevated alkaline phosphatase, and elevated phosphorous level [19]. Interestingly, our patient is an obese Caucasian female with hypoalbuminemia, direct bilirubinemia, and an elevated alkaline phosphatase and phosphorus level. Furthermore, her prolonged shock likely resulted in ischemia induced cellular injury which may have provoked the dedifferentiation of smooth muscle cells of vascular tissue to produce osteopontin creat- 
ing calcification. Additionally, her acute myeloid leukemia may have further aggravated ischemia in small vessels. Leukemic blast cells have been shown to cause an increase in flow resistance and subsequent obstruction for erythrocytes to traverse the microcirculation [20]. Although her renal function was only minimally impaired following the administration of antibiotics and intravenous fluid resuscitation, we note her significant acute kidney injury upon presentation and question if this could have represented the harbinger of her subsequent development of diffuse calciphylaxis.

To date, there are no controlled, prospective studies to help dictate specific, efficacious therapeutic strategies in patients with calciphylaxis. Interventions have included cinacalcet [21], sodium thiosulfate [22], bisphosphonates [23,24], hyperbaric oxygen [25] and correction of underlying plasma calcium and phosphorous concentrations [26]. Although the prognosis of calciphylaxis remains poor, earlier diagnosis portends a more favorable clinical outcome. Therefore, clinicians should keep this rare association in mind when caring for patients with acute myeloid leukemia, as prompt diagnosis and subsequent treatment may alter an otherwise sinister disease process.

\section{CONCLUSION}

This case is unique in that, to our knowledge and review of the literature, calciphylaxis has never been reported in association with acute myeloid leukemia. No clear pathophysiologic processes have been defined that lead to calciphylaxis. Multiple reports suggest it to be a complicated process requiring specific patient characteristics that may increase the likelihood of developing calciphylaxis in the face of certain physiochemical insults. Further research will be important to identify additional predisposing characteristics and general stressors that lead to the development of calciphylaxis. This knowledge will help in both intervention and treatment of this condition going forward. This case will raise awareness in physicians caring for patients with acute myeloid leukemia and this rare association.

\section{REFERENCES}

[1] Selye, H. (1962) The dermatologic implications of stress and calciphylaxis. Journal of Investigative Dermatology, 39, 259-275. doi:10.1038/jid.1962.111

[2] Selye, H. (1962) Calciphylaxis. University of Chicago Press, Chicago.

[3] Selye, H., Gabbiani, G., Strebel, R. (1962) Sensitization to calciphylaxis by endogenous parathyroid hormone. Endocrinology, 71, 554-558. doi:10.1210/endo-71-4-554

[4] Selye, H., Gentile, G., Pioreschi, P. (1961) Cutaneous molt induced by calciphylaxis in the rat. Science, 134, 1876-1877. doi:10.1126/science.134.3493.1876
[5] Coates, T., Kirkland, G.S., Dymock, R.B., Murphy, B.F., Brealey, J.K., Mathew, T.S.H., et al. (1998) Cutaneous necrosis from calcific uremic arteriolopathy. The American Journal of Kidney Diseases, 32, 384-391. doi:10.1053/ajkd.1998.v32.pm9740153

[6] Bleyer, A.J., White, W.L., Choi, M.J. (2000) Calcific small vessel ischemic disease (calciphylaxis) in dialysis patients. International Journal of Artificial Organs, 23, 351-355.

[7] Torok, L. and Kozepessy, L. (1991) Uraemic gangrene syndrome. Acta Dermto Venereologica, 71, 455-457.

[8] Dahl, P.R., Winkelmann, R.K. and Connolly, S.M. (1995) The vascular calcification-cutaneous necrosis syndrome. Journal of the American Academy of Dermatology, 33, 53-58. doi:10.1016/0190-9622(95)90010-1

[9] Weenig, R.H. (2008) Pathogenesis of calciphylaxis: Hans Selye to nuclear factor $\mathrm{K}-\mathrm{B}$. Journal of the American Academy of Dermatology, 58, 458-471. doi:10.1016/j.jaad.2007.12.006

[10] Weenig, R.H., Sewell, L.D., Davis, M.D.P., McCarthy, J.T. and Pittelkow, M.R. (2007) Calciphylaxis: Natural history, risk factor analysis, and outcome. Journal of the American Academy of Dermatology, 56, 569-579. doi:10.1016/j.jaad.2006.08.065

[11] Nigwekar, S.U., Wolf, M., Sterns, R.H. and Hix, J.K. (2008) Calciphylaxis from nonuremic causes: A systematic review. Clinical Journal of the American Society of Nephrology, 3, 1139-1143. doi:10.2215/CJN.00530108

[12] Mastruserio, D.N., Nguyen, E.Q., Nielsen, T., Hessel, A. and Pellegrini, A.E. (1999) Calciphylaxis associated with metastatic breast carcinoma. Journal of the American Academy of Dermatology, 41, 295-298. doi:10.1016/S0190-9622(99)70368-3

[13] Raper, R.F., Ibels, L.S. (1985) Osteosclerotic myeloma complicated by diffuse arteritis, vascular calcification and extensive cutaneous necrosis. Nephron, 39, 389-392. doi: $10.1159 / 000183411$

[14] Sibai, H., Ishak, R.S., Halawi, R., et al. (2012) Non-uremic calcific arteriolopathy (calciphylaxis) in relapsed/ refractory Hogkin's lymphoma: A previously unreported association. Journal of Clinical Oncology, 30, e88-e90. doi:10.1200/JCO.2011.39.4551

[15] Goff, H.W. and Grimwood, R.E. (2005) A case of calciphylaxis and chronic myelomonocytic leukemia. Cutis, 75, 325-328.

[16] Riegert-Johnson, D.L., Kaur, J.S. and Pfeifer, E.A. (2001) Calciphylaxis associated with cholangiocarcinoma treated with low- molecular-weight heparin and vitamin K. Mayo Clinic Proceedings, 76, 749-752. doi:10.4065/76.7.749

[17] Kutlu, N.O., Aydin, N.E., Aslan, M., et al. (2003) Malignant melanoma of the soft parts showing calciphylaxis. Pediatric Hematology Oncology, 20, 141-146. doi:10.1080/0880010390158621

[18] Ahmed, S., O’Neill, K.D., Hood, A.F., Evan, A.P. and Moe, S.M. (2001) Calciphylaxis is associated with hyperphosphatemia and increased osteopontin expression by vascular smooth muscle cells. The American Journal of Kidney Diseases, 37, 1267-1276. doi:10.1053/ajkd.2001.24533 
[19] Kalajian, A.H., Malhotra, P.S., Callen, J.P., et al. (2009) Calciphylaxis with normal renal and parathyroid function: Not as rare as previously believed. Archives of Dermatology, 145, 451-458. doi:10.1001/archdermatol.2008.602

[20] LaCelle, P.L. (1986) Alterations by leukocytes of erythrocyte flow in microchannels. Blood Cells, 12, 179-189.

[21] Robinson, M.R., Augustine, J.J. and Korman, N.J. (2007) Cinacalcet for the treatment of calciphylaxis. Archives of Dermatology, 143, 152-154. doi:10.1001/archderm.143.2.152

[22] Cicone, J.S., Petronis, J.B., Embert, C.D. and Spector, D.A. (2004) Successful treatment of calciphylaxis with intravenous sodium thiosulfate. The American Journal of Kidney Diseases, 43, 1104-1108. doi:10.1053/j.ajkd.2004.03.018

[23] Shiraishi, N., Kitamura, K., Miyoshi, T., et al. (2006) Successful treatment of a patient with severe calcific uremic arteriolopathy (calciphylaxis) by etidronate disodium. The American Journal of Kidney Diseases, 48, 151-154. doi:10.1053/j.ajkd.2006.04.062

[24] Monney, P., Nguyen, Q.V., Perroud, H. and Descombes, E. (2004) Rapid improvement of calciphylaxis after intravenous pamidronate therapy in a patient with chronic renal failure. Nephrology Dialysis Transplantation, 19, 2130-2132. doi:10.1093/ndt/gfh305

[25] Podymow, T., Wherrett, C. and Burns, K.D. (2001) Hyperbaric oxygen in the treatment of calciphylaxis: A case series. Nephrology Dialysis Transplantation, 16, 21762180. doi:10.1093/ndt/16.11.2176

[26] Block, G.A. (2000) Prevalence and clinical consequences of elevated $\mathrm{Ca} \times \mathrm{P}$ product in hemodialysis patients. Clinical Nephrology, 54, 318-324. 